\title{
Polarization stable hollow core fiber interferometer with Faraday rotator mirrors
}

\author{
Radan Slavík, Senior member, IEEE, Daniel Dousek, Dmytro Suslov, Matěj Komanec, Stanislav \\ Zvánovec, Francesco Poletti, and David J. Richardson, Fellow IEEE
}

\begin{abstract}
We report the fabrication of a hollow core fiber (HCF) coupled Faraday rotator mirror component with a total insertion loss below $1.1 \mathrm{~dB}$ and demonstrate its use in a polarization-stable all-fiber Michelson interferometer. The signal split into the two interferometer arms is performed using a standard $2 \times 2$ fused fiber coupler. One of the coupler output ports is permanently and directly connected to the HCF with a low return loss (below $-40 \mathrm{~dB}$ ) and negligible signal coupling into higher-order modes. The demonstrated polarization-insensitive interferometer shows residual peak-to-peak polarization-induced power variations as low as $0.04 \mathrm{~dB}$. The device will be of interest in interferometric applications requiring low thermal sensitivity as the phase drift of light propagating through an HCF is over an order of magnitude less sensitive to temperature than in standard fiber. Moreover, the fiber nonlinearity is almost three orders of magnitude lower in HCFs as compared to standard solid optical fibers.
\end{abstract}

Index Terms-Hollow core fibers, fiber interferometers

\section{INTRODUCTION}

$\mathrm{F}$ IBER-based interferometers are popular in fields such as sensing [1,2] and metrology [3]. Optical fibers together with fiber couplers allow for the construction of alignment-free interferometers in which each interferometer arm can be meters to kilometers long thanks to the low attenuation of optical fibers and the fact that they can be readily and compactly coiled. However, optical fibers suffer from small randomly varying birefringence along their length due to fabrication imperfections [4] and bend-induced birefringence [5]. This birefringence changes with temperature and fiber bending/twisting. This has two undesired consequences. Firstly, environmentally induced changes in the polarization of light propagating through the fiber can negatively impact the quality of interference between the two interfering signals, commonly referred to as "polarization signal fading." Secondly, a changing temperature, and/or bending, can result in random coupling of light between the slow and fast axes of the optical fiber. Consequently, the phase of light propagating through the fiber changes with these external fiber perturbations, reducing the stability of the interference pattern. Finally, the effective optical path length $n_{\text {eff: }} L$ and thus the phase of the propagating light, changes with temperature due to the thermo-optic effect and thermal expansion of the fiber core glass [1]. Here $n_{\text {eff }}$ is the fiber mode effective refractive index and $L$ is the physical fiber length [1]. This phase causes the interference pattern to drift with temperature.

The polarization signal fading and optical path length variations due to random fiber birefringence can be mitigated either by using polarization-maintaining (PM) fibers or by using standard fibers in the Michelson interferometer with $90^{\circ}$ Faraday rotator mirrors (FRMs) placed at the fiber ends [6], see Fig. 1. In this configuration, the light propagates through the fiber twice and in such a way that it has an orthogonal polarization in the backward direction relative to the forward direction at all points along the fiber length. This ensures that the light always propagates equally through both fiber polarization eigenstates over the full round trip, meaning that the path length is insensitive to the fiber birefringence. The other advantage is that the light always has a fixed polarization state after the round-trip propagation through the fiber, ensuring stable interference contrast, even when the fiber birefringence is changing, e.g., due to touching of the fiber in the interferometer arms. The FRM configuration is especially preferred over the use of PM fibers when long fiber lengths are needed since fiber-coupled FRMs currently cost about the same as $30 \mathrm{~m}$ or so of PM fiber. However, it may be advantageous to use FRMs even for short fiber lengths, e.g., due to the practical limits of polarization axis alignment associated with splicing PM fibers, or due to the relatively high cost of PM fiber components that may be needed inside the interferometer, e.g., fiber phase shifters, etc.

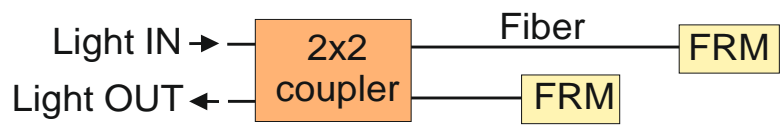

Fig. 1. Schematic of the polarization-stable Michelson fiber interferometer with Faraday rotator mirrors (FRMs).

R. Slavík, F. Poletti, and D. J. Richardson are with the Optoelectronics Research Centre, University of Southampton, Southampton SO17 1BJ, U.K (e-mail: r.slavik@soton.ac.uk)

M. Komanec, D. Dousek, D. Suslov, and S. Zvánovec are with the Faculty of Electrical Engineering, Czech Technical University in Prague, 16627 Prague, Czech Republic.
Manuscript received July 5, 2021; This work was supported in part by the Royal Academy of Engineering (RCSFR1718\6\15), and in part by the Engineering and Physical Sciences Research Council (EPSRC) under Grant EP/P030181/1, and in part by the Czech Technical University (CTU) in Prague under Grant SGS20/166/OHK3/3T/13. (Corresponding author: $R$ Slavík) 
The third effect (temperature-dependent fiber optical path length) is, however, more challenging to address. In applications where an interferometer with equal fiber paths in both arms can be used, this can be addressed to a certain degree by using a Sagnac loop $[7,8]$. However, in most applications, the path lengths need to be different to create a large delay, e.g., in a delay-line interferometer for laser stabilization [3], or if access to only one interferometer arm is desired, e.g., for fiber sensing [1], where often the desire is that the sensing event influences only one interferometer arm, whilst the other serves as a reference. For these applications, thermal sensitivity causes unwanted effects and generally limits performance. It is usually addressed via engineering approaches, e.g., by subtracting the changes due to the temperature or by stabilizing the optical path lengths of the interferometer [10]. It can also be improved via passive means such as temperature stabilization, or by the use of fibers that have a lower sensitivity to temperature $[11,12]$.

In particular, Hollow Core Fibers (HCF) have been shown to have a thermal sensitivity that is more than an order of magnitude lower than standard single-mode fibers (SSMFs) [13]. Although HCF can be permanently connected with SSMF with low loss (0.15 $\mathrm{dB}$ [15]) and low back-reflection $(<-40 \mathrm{~dB}$ [15]), their use in an FRM-assisted Michelson interferometer has not yet been demonstrated.

In this paper, we demonstrate an FRM-assisted Michelson interferometer based on HCF. To minimize the insertion loss as well as unwanted coupling into the HCF higher-order modes that may cause multi-path interference, we interconnect the FRM directly to the HCF. We show that this approach leads to an interferometer with minimal multi-path interference degradation and allows for a robust HCF-based fiber interferometer to be built. It should be of interest in applications where a low thermal sensitivity is needed, or when an interferometer with two arms made of optical fibers with significantly different thermal, acoustic, bending, or other sensitivities is desired.

\section{DEVICE ASSEMBLY}

The FRM-HCF was fabricated in two steps, Fig. 2. Firstly, Fig. 2a, we glued together a mirror (protected silver mirror, $7 \mathrm{~mm}$ diameter, Thorlabs, model PF03-03-P01), a $45^{\circ}$ Faraday rotator that consisted of a $2 \times 2 \mathrm{~mm}^{2}$ crystal enclosed within a circular magnet housing (diameter $3 \mathrm{~mm}$, clear aperture $0.9 \mathrm{~mm}$, Ideal Photonics, model FSFR-1550-09-30x30), and an AR-coated graded index (GRIN) collimating lens (1.8-mm diameter, Thorlabs, model GRIN2915) inserted into a tube (Thorlabs, 512800-1800) that was shortened to approximately the same length as the GRIN collimating lens. The tube effectively increased the diameter of the GRIN lens to $2.8 \mathrm{~mm}$, allowing for easy gluing of all three components without getting the glue into the light path. The three components were passively aligned and glued with epoxy.

In the next step, Fig. 2b, we prepared the HCF end facet. We used a Nested Antiresonant Nodeless Fiber (NANF) type of $\mathrm{HCF}$ - the same sample as in [15]. It operated in the $2^{\text {nd }}$ antiresonant window at $1550 \mathrm{~nm}$, with the inner structure consisting of six large tubes with smaller tubes nested inside them, and a core diameter of $32.5 \mu \mathrm{m}$. Its end face image is shown as an inset in Fig. 2b. We followed the same procedure for $\mathrm{HCF}$ preparation as for the SSMF-HCF interconnect reported in Ref. [14,15]. The front facet (entitled 'Glass block') of the assembly is shown as an inset in Fig. 2b. To prepare it, we glued two V-grooved glass half-blocks together, making an empty fiber-array glass block of about $1 \times 3 \mathrm{~mm}^{2}$ cross-section (dimension comparable to that of the GRIN-in-tube diameter to which we glued it later on) with an inner hole formed by the two opposing V-groves that was just slightly larger than the diameter of the HCF used. Subsequently, we cleaved the HCF, inserted it into the inner hole, and glued it in place using an UVcurable glue.

(a) Step 1: Passive alignment \& glueing

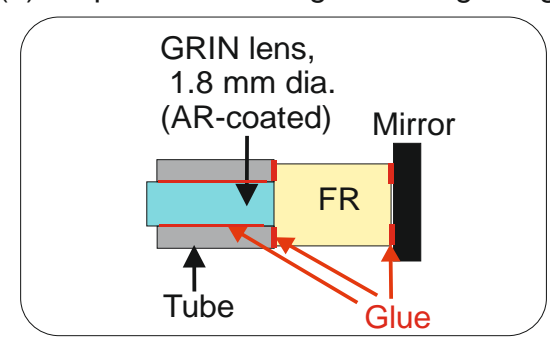

(b) Step 2: Active alignment \& glueing

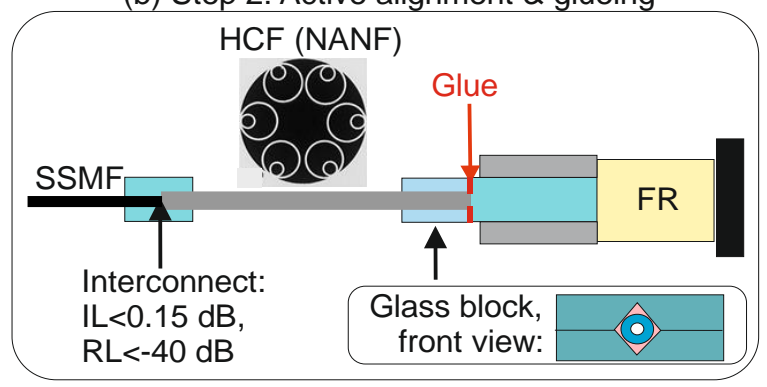

Fig. 2. (a) Passively aligned and subsequently glued together assembly comprising: a mirror, $45^{\circ}$ free-space miniature Faraday rotator (FR), and ARcoated 1/4-pitch GRIN lens put in a tube of a similar diameter to the FR. The GRIN lens serves as a collimator. (b) Subsequently, an HCF with its core structure shown as an inset with permanent high-performance interconnection to SSMF [15] is put through a hole in a glass block made of two opposing Vgroves (inset), actively-aligned to the assembly shown in (a), and glued together.

In the last step, we actively aligned this HCF assembly with the FRM assembly using a broadband light source (amplified spontaneous emission from an erbium-doped fiber amplifier, EDFA) and $\mathrm{x}, \mathrm{y}, \mathrm{z}$, pitch and yaw 5-D stages while monitoring the insertion loss and higher order mode content using the optical spectrum analysis method described in reference [15]. After the alignment, which targets the lowest insertion loss and lowest coupling into the higher order modes, we applied a UVcurable glue around the two sub-assemblies while taking care that the glue does not seep inside the HCF hole, and then cured it with a UV lamp. 


\section{DEVICE CHARACTERIZATION}

The performance of an FRM is best characterized using a polarization beam splitter, as shown in Fig. 3. Here, we use a fiber-coupled polarization beam splitter, which has the common 'input' (COM port) made of SSMF and two output ports that collect the s-polarized and p-polarized light components with two PM fibers aligned to the slow axes. We launched light into one of the PM fibers and connected the SSMF COM port to our SSMF-HCF-FRM assembly. The light propagates along the fiber (SSMF pigtail and then through the $\mathrm{HCF}$ ) and gets reflected back with its polarization always $90^{\circ}$ rotated with respect to the forward-propagating light. The light at the polarization beam splitter is then coupled into the other PM port, as the light coming back should have the orthogonal polarization with respect to the forward-propagating light. Indeed, the operation should be independent of the polarization changes induced in the reflection-operated fiber, which is why we include a polarization controller there (Fig. 3).

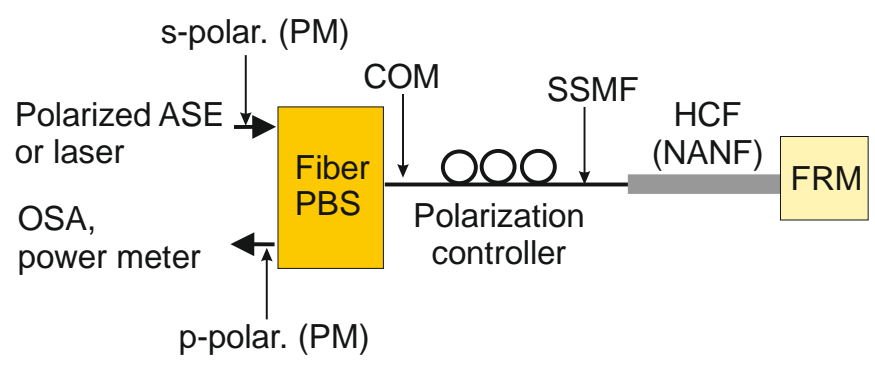

Fig. 3. Characterization of the SMF-HCF-FRM assembly using an all-fiber polarization beam splitter (PBS) with a common SSMF-pigtailed port (COM) from which light is split into s-polarization and p-polarization (both ports pigtailed with a PM fiber).

As with any HCF-based system, it is important to ensure that operation is not degraded by the back-reflections that may occur at an SSMF-HCF interface due to the 3.5\% Fresnel backreflection as light travels from the glass material to air inside the HCF core. As previously mentioned, we suppressed this back-reflection to below $-40 \mathrm{~dB}$ by applying an AR coating to the interface, which we described in our previous work [15].

The other phenomenon that one needs to consider when working with HCFs that are not truly single-moded is the potential impact of undesired coupling into higher order HCF modes. This can again take place at the interfaces, i.e., SSMFHCF interface and HCF-FRM interface. We have actively controlled this during assembly, as described in detail in [15]. The cross-coupling into higher order modes is not a problem on its own. However, it becomes a problem when the light from a higher order mode is coupled back into the fundamental mode, as this creates multi-path interference. As higher order modes have slightly different propagation constants to the fundamental mode, it manifests itself as a periodic power variation in the optical spectrum.

Fig. 4 shows the transmission spectrum of our SSMF-HCFFRM assembly. To account for the spectral characteristic of the $\mathrm{HCF}$ and the polarization beam splitter transmission characteristics, we referenced it by replacing the fabricated
SSMF-HCF-FRM assembly in the set-up shown in Fig. 3 with an SSMF-FRM of known insertion loss. The insertion loss of $1.4 \mathrm{~dB}$ (Fig. 4) includes also the loss at the SSMF-HCF interface $(0.15 \mathrm{~dB})$ through which the light passes twice, resulting in an insertion loss for the HCF-FRM component of $1.1 \mathrm{~dB}$. The spectral oscillations (the most visible has a period of about $0.25 \mathrm{~nm}$ ) are due to multi-path interference with higher order modes excited at the HCF interfaces. These are extremely low in our assembly - causing a peak-to-peak power variation of only $0.04 \mathrm{~dB}$, thus showing negligible influence of multipath interference on performance. We also evaluated the polarization properties by changing the polarization through the polarization controller (Fig. 3). The characteristics in terms of the insertion loss or amplitude of the spectral loss variations did not change within the measurement error, suggesting correct operation of the assembly.

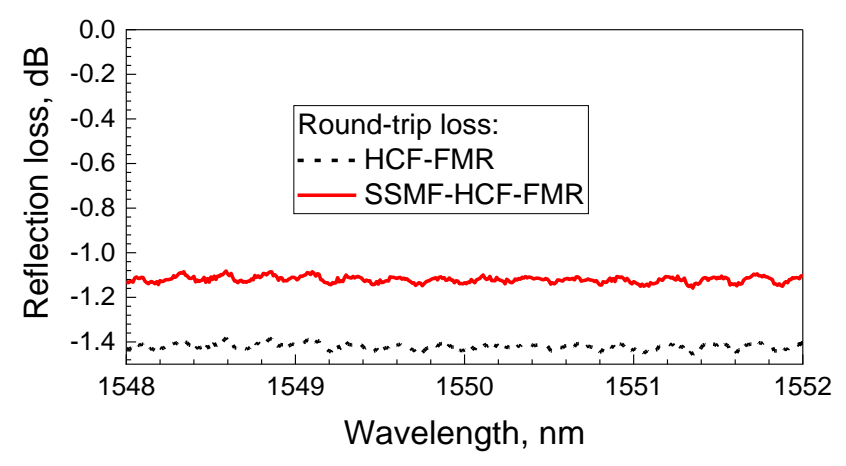

Fig. 4. Reflection spectrum of the HCF-FRM and the entire SSMF-HCF-FRM assembly.

\section{POLARIZATION INSENSITIVE INTERFEROMETER}

We envisage two key interferometer configurations that can take advantage of the key HCF properties of low thermal sensitivity that can be exploited to make the interferometer less sensitive to ambient temperature variations and/or low nonlinearity. The first one is a classical delay line interferometer in which one arm is long (made of HCF) and the other one is as short as possible (made most probably from a SSMF-FRM). The second interferometer configuration would have HCF in both arms.

In this letter, we demonstrate the interferometer performance in terms of its low thermal sensitivity. We built a Michelson interferometer, as shown in Fig. 5, where one interferometer arm consists of a 50-cm long SSMF $2 \times 2$ coupler pigtail followed by the $7.5-\mathrm{m}$ long HCF-FRM, while the other arm consists of equal length of the SSMF pigtail $(50 \mathrm{~cm})$ followed by a commercially-available SSMF-coupled FRM. The SSMFs in both arms have the same length to within $2 \mathrm{~cm}$, and we placed them in close proximity to ensure they experience as identical a thermal environment as possible. Subsequently, we put the entire interferometer into a thermal chamber. We interrogated it with a stable CW laser (Rock from NP Photonics) and monitored the output power with a photodiode. We increased the temperature in $3^{\circ} \mathrm{C}$ steps and counted how many times the output comes through constructive interference. Each count corresponds to an interferometer phase 
change of $2 \pi$. The result is shown in Fig. 6 . Here we see that the interferometer phase changes at a rate of $28 \mathrm{rad} /{ }^{\circ} \mathrm{C}$. Considering interferometer imbalance of $15 \mathrm{~m}$ (7.5 m of HCF), the thermal sensitivity is calculated to be $1.9 \mathrm{rad} / \mathrm{m} /{ }^{\circ} \mathrm{C}$. This agrees well with the value measured for $\mathrm{HCF}$, which is about 20 times lower than the value reported for SSMF in an SSMFbased interferometer [13].

The interference contrast was measured to be 25-26 dB (as shown in insert in Fig. 6) and as expected, did not change when the input polarization was altered. This value, which is sufficient for most applications, was most likely limited by the differential loss in both interferometer arms.

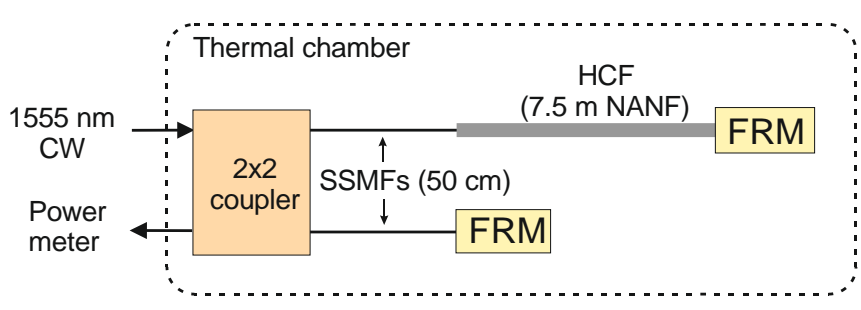

Fig. 5. Experimental set-up to measure thermal sensitivity of the fabricated polarization-insensitive interferometer with one arm made of HCF.

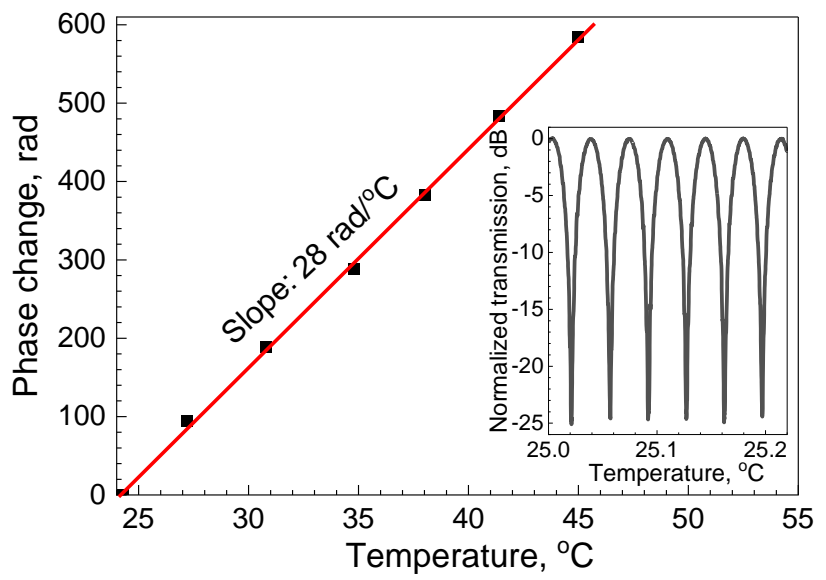

Fig. 6. Phase change in Michelson interferometer shown in Fig. 5 with temperature and its linear fit. Inset shows measured power when temperature was changing, showing interference contrast over $25 \mathrm{~dB}$. HCF was $7.5 \mathrm{~m}$ long.

\section{CONCLUSIONS}

We have demonstrated an alignment-free (once fabricated) all-fiber polarization-stable Michelson interferometer incorporating an HCF. The key expected advantages of using HCF as compared to SSMF stems from their order of magnitude lower sensitivity to temperature [13] and almost three orders of magnitude higher non-linear threshold [16]. The HCF used (NANF type) also provides an added benefit of almost an order of magnitude lower fiber chromatic dispersion. Firstly, we built a packaged HCF-coupled Faraday rotator mirror with an insertion loss below $1.1 \mathrm{~dB}$. We then characterized the thermal stability of the Michelson interferometer and showed that it is as low as expected from the low thermal sensitivity of the HCF.
The interference contrast was $>25 \mathrm{~dB}$, achieved even when deliberately manually perturbing the fibers. Further, the power variations due to higher order mode excitation in the HCF were measured to be as low as $0.04 \mathrm{~dB}$. The demonstrated HCFbased FRM and Michelson interferometer will be of interest for fiber optic sensors, metrology, and quantum devices/systems.

\section{ACKNOWLEDGEMENT}

The data for this work is accessible through the University of Southampton Institutional Research Repository (DOI: https://doi.org/10.5258/SOTON/D2035).

\section{REFERENCES}

1. B.H. Lee, Y.H. Kim, K.S. Park, J.B. Eom, M.J. Kim, B.S. Rho, and H.Y. Choi, "Interferometric fiber optic sensors," Sensors, vol. 12, pp. 24672486, Feb. 2012, DOI. 10.3390/s120302467.

2. B. Culshaw and A. Kersey, Fiber-Optic Sensiting: A historical perspective, IEEE J. of Lightwave Technol., vol. 26, no. 9, pp. 1064-1078, May 2008.

3. F. Kéfélian, H. Jiang, P. Lemonde, and G. Santarelli, "Ultralowfrequency-noise stabilization of a laser by locking to an optical fiber-delay line," Opt. Lett. vol. 34, no. 7, pp. 914-916, Apr. 2009.

4. S. Rashleigh, "Origins and control of polarization effects in single-mode fibers," Origins and control of polarization effects in single-mode fibers," IEEE J. of Lightwave Techol., vol. LT-1, no. 2, pp. 312-331, June 1983.

5. R. Ulrich, S. Rashleigh, and W. Eickhoff, "Bending-induced birefringence in single-mode fibers," ," Opt. Lett., vol. 5, no. 6, pp. 273275, June 1980 .

6. A.D. Kersey, M.J. Marrone, and M.A. Davis, "Polarisation-insensitive fibre optic Michelson interferometer, Electron. Lett., vol. 27, no. 6, pp. 518-520, March 1991.

7. S. Blin, H.K. Kim, M.J.F. Digonnet, and G.S. Kino, "Reduced thermal sensitivity of a fiber-optic gyroscope using an air-core photonics-bandgap fiber," IEEE J. of Lightwave Technol., vol. 25, no. 3, pp. 861-865, March 2007.

8. D.M. Shupe, "Thermally induced nonreciprocity in the fiber-optic interferometer," Appl. Opt., vol. 19, no. 5, pp. 654-655, 1980.

9. V. Vali and R.W. Shorthill, "Fiber ring interferometer," Appl. Opt., vol 15, no. 5, pp. 1099-1100, May 1976.

10. S.B. Cho and T.G. Noh, "Stabilization of a long-armed fiber-optic singlephoton interferometer," Opt. Express, vol. 17, no. 21, pp. 19027-19032, Oct 2009.

11. M. Bousonville et al, "New phase stable optical fiber," in Proc. Beam Instrum. Workshop, 2012, pp. 101-103.

12. V. Dangui, H.K. Kim, M.J.F. Digonnet, and G.S. Kino, "Phase sensitivity to temperature of the fundamental mode in air-guiding photonic-bandgap fibers," Opt. Exp., vol. 13, no.18, pp. 6669 - 6684, July 2005.

13. R. Slavík, G. Marra, E. N. Fokoua, N. Baddela, N. V. Wheeler, M. Petrovich, F. Poletti and D. J. Richardson, "Ultralow thermal sensitivity of phase and propagation delay in hollow core optical fibres," Sci. Rep., vol. 5, article 15447, 2015.

14. M. Komanec, D. Suslov, S. Zvánovec, Y. Chen, T. Bradley, S.R. Sandoghchi, E.R. Numkam Fokoua, G.T. Jasion, M.N. Petrovich, F. Poletti, D.J. Richardson, and R. Slavík, "Low-loss and low-backreflection hollow-core to standard fiber interconnection," IEEE Photon. Tech. Lett, vol. 31, vo. 10, pp. 723-726, May 2019.

15. D. Suslov, M. Komanec, E.R. Numkam Fokoua, D. Dousek, A. Zhong, S. Zvánovec, T. Bradley, F. Poletti, D.J. Richardson, and R. Slavík, "Low loss, high-performance interconnection between standard single-mode fiber and antiresonant hollow-core fiber", early access Sci. Rep. 2021. DOI. 10.1038/s41598-021-88065-2

16. M. Michieletto, J.K. Lyngsø, Ch. Jakobsen, J. Lægsgaard, O. Bang, and T.T. Alkeskjold, "Hollow-core fibers for high power pulse delivery", Opt. Express, vol. 24, no. 7, pp. 7103-7119, Apr. 2016 doi:10.1364/OE.24.007103. 